\title{
Oral rehabilitation of Papillon-Lefèvre syndrome patients by dental implants: a systematic review
}

\author{
Fazele Atarbashi-Moghadam ${ }^{1}$, Saede Atarbashi-Moghadam², Setare Kazemifard ${ }^{3}$, \\ Soran Sijanivandi ${ }^{3}$, Mahshid Namdari ${ }^{4,5}$ \\ ${ }^{1}$ Department of Periodontics, Dental School of Shahid Beheshti University of Medical Sciences, \\ ${ }^{2}$ Department of Oral and Maxillofacial Pathology, School of Dentistry, Shahid Beheshti University of Medical Sciences, \\ ${ }^{3}$ Dental Research Center, Research Institute of Dental Sciences, Shahid Beheshti University of Medical Sciences, \\ ${ }^{4}$ Community Oral Health Department, School of Dentistry, Shahid Beheshti University of Medical Sciences, \\ ${ }^{5}$ Department of Biostatistics, School of Allied Medical Sciences, Shahid Beheshti University of Medical Sciences, Tehran, Iran
}

\begin{abstract}
J Korean Assoc Oral Maxillofac Surg 2020;46:220-227)
Objectives: Papillon-Lefèvre syndrome (PLS) is a rare autosomal recessive disorder. These patients lose their teeth at a young age and are in need of prosthetic rehabilitation. The aim of this systematic review was to assess the success of dental implant placement in these patients.

Materials and Methods: An electronic search was performed in PubMed Central, Scopus, and Web of Science using the keyword "Papillon-Lefèvre syndrome" AND "dental implant" OR "prosthodontics". Articles reporting implant placement in patients with PLS until July 2019 were included.

Results: Assessment of the included 11 articles reporting 15 cases showed 136 implant placements in these patients. Implant failure occurred in 3 patients (20 implants). The peri-implantitis and failure rate was higher in the maxilla. Meta-analysis showed the probability of failure to be $7 \%(95 \%$ confidence interval $[\mathrm{CI}] 0 \%-31 \%$ ) for maxillary implants and $2 \%(95 \%$ CI $0 \%-9 \%)$ for mandibular implants. The follow-up time ranged between 1 and 20 years. Healing after bone graft and implant placement in these patients was uneventful.

Conclusion: Dental implants may be a viable treatment option for PLS patients. Implantation can help preserve alveolar bone if the patients' immunological and growing conditions are well-considered and proper oral hygiene and compliance with the maintenance program are continued.
\end{abstract}

Key words: Papillon-Lefèvre syndrome, Dental implants, Oral rehabilitation, Prosthodontics, Rapid progressive periodontitis

[paper submitted 2020. 3. 4 / revised 2020. 6. 5 / accepted 2020. 6. 5]

\section{Introduction}

Full or partial edentulism in children and adolescents occurs most commonly as a result of trauma or genetic disorders, and these patients encounter esthetic and functional problems ${ }^{1}$. Esthetic problems can cause psychologic disorders and affect social activity ${ }^{1,2}$. Long-standing edentulism leads to a senile profile and a decreased facial height ${ }^{3}$. The functional problems experienced include difficulty in eating and speak-

\section{Soran Sijanivandi}

Dental Research Center, Research Institute of Dental Sciences, Shahid Beheshti University of Medical Sciences, Tehran 1983969411, Iran

TEL: +98-920-618-3943 FAX: +98-21-44424248

E-mail:s.r.sijanivandi@gmail.com

ORCID: https://orcid.org/0000-0003-4663-4423

(c) This is an open-access article distributed under the terms of the Creative Commons Attribution Non-Commercial License (http://creativecommons.org/ licenses/by-nc/4.0/), which permits unrestricted non-commercial use, distribution, and reproduction in any medium, provided the original work is properly cited.

Copyright (C) 2020 The Korean Association of Oral and Maxillofacial Surgeons. All rights reserved. ing $^{1,2}$. Papillon-Lefèvre syndrome (PLS; OMIM\#245000) is one of the disorders that can cause early tooth loss ${ }^{3,4}$, and those affected by PLS usually need oral rehabilitation to overcome these problems ${ }^{3}$.

PLS is a rare autosomal recessive disorder with dermal and oral manifestations ${ }^{4,5}$. Mutation in the cathepsin $\mathrm{C}$ gene (CTSC), which is localized on chromosome 11q14-q21, in these patients causes the loss of cathepsin $\mathrm{C}$ function. $\mathrm{Ca}-$ thepsin $\mathrm{C}$ plays a role in epithelial differentiation and desquamation ${ }^{6}$. The severity of dermal manifestations, including diffuse hyperkeratosis affecting the palms of the hands and soles of the feet, varies in patients from mild psoriasiform scaly skin to overt hyperkeratosis ${ }^{4,5}$. The soles of the feet are usually affected more than the palms, and the degree of hyperkeratosis may be affected by seasonal changes. There is evidence that the hyperkeratosis severity is associated with periodontal involvement ${ }^{5}$. PLS patients' neutrophil functions are affected, and these dysfunctions appear to be localized to 
tissues that are under direct and chronic bacterial attacks like the periodontal tissue. This has been suggested to be the possible reason for PLS patients' lack of systemic infections ${ }^{7}$.

Rapid progressive destructive periodontitis around primary and permanent dentitions is the oral manifestation of PLS ${ }^{8}$. The inflammatory gingival changes and periodontitis around primary dentition appear concomitant with palmoplantar keratosis and subside after tooth $\operatorname{loss}^{5}$. Because of this, almost all PLS patients lose their teeth at a young age. Surprisingly, third molars, which erupt at older ages, are not affected ${ }^{3,5}$. PLS patients' immune system function improves with age and their polymorphonuclear (PMN) cells' chemotaxis and phagocytosis abilities become normal after some years of being edentulous ${ }^{8,9}$.

Dental implants can be a viable treatment option for these patients in order to overcome esthetic and functional prosthetic problems ${ }^{10}$. However, issues surrounding implantation raise success concerns in these patients; these issues include impaired patient immune systems and severe bone loss that results in the need for complex ridge augmentations ${ }^{10,11}$. The aim of this systematic review was to assess the clinical outcome and the survival rate of dental implants used for the oral rehabilitation of PLS patients.

\section{Materials and Methods}

The present study is a systematic review that was performed in accordance with the "Preferred Reporting Items for Systematic Review and Meta-Analyses Protocols" (PRISMA$\mathrm{P}, 2015)^{12}$. The search was performed using "MeSH" terms and keywords based on the elements of the PICO question:

1. Participants (P): PLS patients

2. Intervention (I): Oral rehabilitation using dental implants

3. Comparison or control (C): Not applicable

4. Outcome measures $(\mathrm{O})$ : Success of dental implant

\section{Information sources and search strategy}

An exhaustive search of the literature available in PubMed Central, Scopus, and Web of Science's electronic databases until July 2019 was conducted. The following keywords were used in the search strategy: "Papillon-Lefèvre syndrome" AND "dental implant" OR "prosthodontics". The full title and abstract of each article were screened by two independent authors (F.A. and S.K.) using predetermined inclusion and exclusion criteria.(Fig. 1) All of the references were selected from the EndNote X9 (Thomson Reuters, Philadelphia, PA, USA). In order to ascertain whether any relevant studies were neglected in the initial search, the bibliographies of the
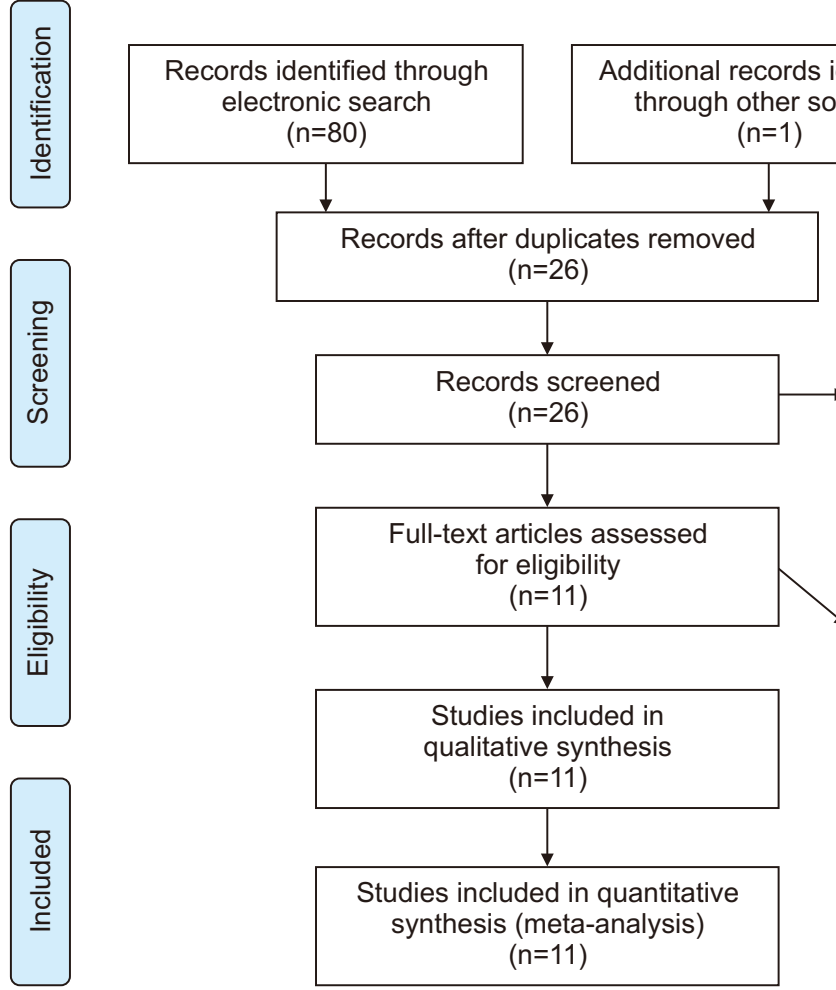

Records excluded $(n=10)$ Reasons:

- Not concerning implants in PLS patients - A 1996 study was excluded due to repetition of results in the follow up study on 2004

Full-text articles excluded $(n=2)$ Reasons: No implant placements
Fig. 1. Diagram of literature search and selection criteria adapted from PRISMA (Preferred Reporting Items for Systematic Reviews and Meta-Analyses) ${ }^{12}$. (PLS: Papillon-Lefèvre syndrome) Fazele Atarbashi-Moghadam et al: Oral rehabilitation of Papillon-Lefève syndrome patients by dental implants: a systematic review. J Korean Assoc Oral Maxillofac Surg 2020 


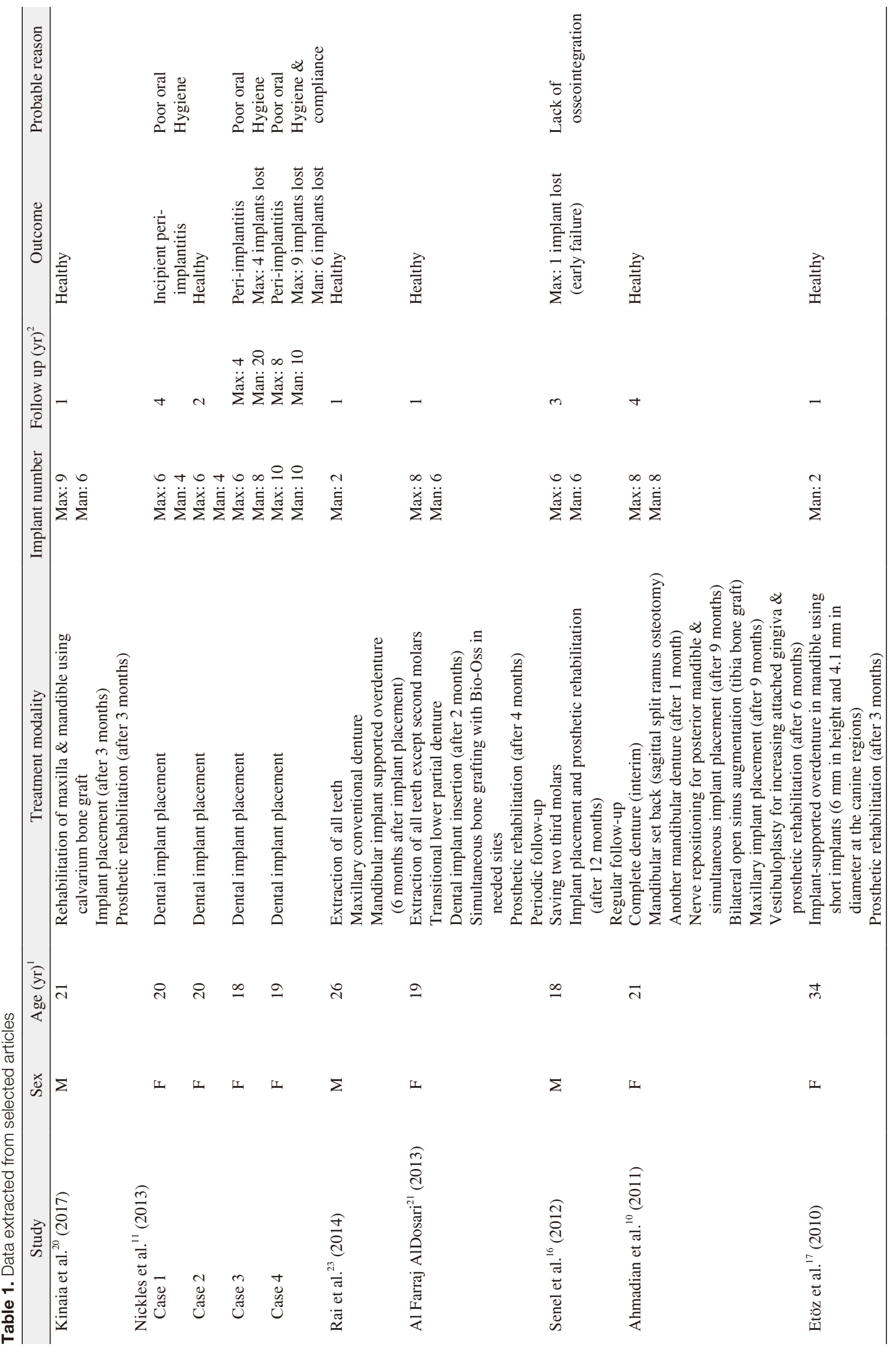




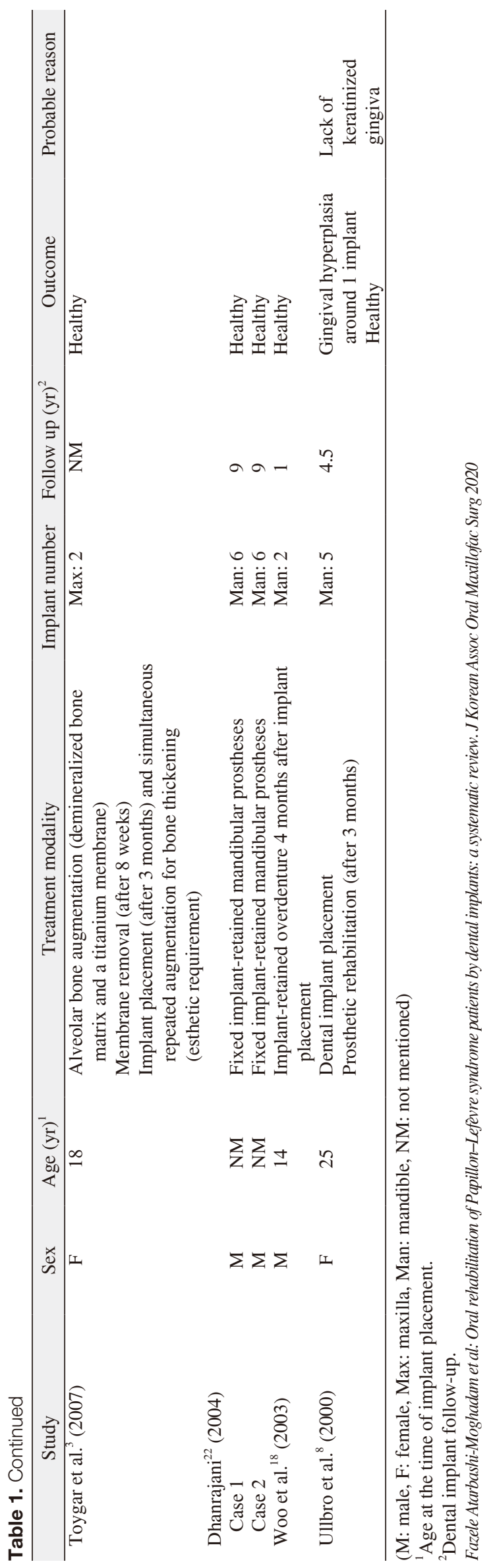

selected studies and Google Scholar were also reviewed. Any differences in the selection of the studies were resolved by discussion with a third reviewer (S.A.).

\section{Eligibility criteria}

Inclusion criteria were articles written in English with full text availability that reported on oral rehabilitation of PLS patients with dental implants, including, for example, case reports and case series.

Exclusion criteria were articles on other prosthetic rehabilitation methods besides dental implants or articles without treatment outcome.

\section{Data collection}

Data were extracted from the included articles by two independent authors (F.A. and S.K.). A third author resolved any disagreements in the extracted data. Microsoft Excel 2016 (Microsoft, Redmond, WA, USA) was used for standardization and organization of the extracted data.

The following information was collected (if available) and is provided in Table 1:

1. Patients' sex and age at the time of implant placement

2. Treatment modality, additional procedures, and grafting donor site or material

3. The number of inserted implants and the outcome of treatment (for example, implant failure, peri-implantitis, or healthy implant)

4. The probable reason for peri-implant diseases

5. The follow-up period after implant placement

6. General characteristics of the selected studies

\section{Quality assessment of studies}

The quality of each study was assessed using CARE (CAse REport) guidelines ${ }^{13}$ to estimate risk of bias. The quality of all thirteen parts was considered.

\section{Statistical method}

Data analysis was performed using Stata statistical software (release 15; StataCorp, College Station, TX, USA). Descriptive data were expressed as percentages. In order to examine the statistical heterogeneity of the data, the $\mathrm{I}^{2}$ statistic was used. To analyze and integrate the results, random and fixed effect methods were used for maxillary and mandibular 
implants, respectively.

\section{Results}

The primary electronic search resulted in 80 studies. After exclusion of irrelevant studies, 11 articles with 15 cases were included in this systematic review.(Table 1) These cases included six male and nine female patients afflicted by PLS who were treated by dental implants. The mean age of patients at the time of implant placement was $20.69 \pm 4.96$ years, and most of implant placement was done after the age 18. A total number of 136 dental implant placements were reported for these patients. Most of the treatment plans called for full mouth rehabilitation followed by mandibular overdenture. One case reported two single implant placements of maxillary central incisors, the only maxillary teeth lost. The patient was under meticulous follow-up before and during permanent teeth eruption. Her mandibular incisors were planned for future reconstruction ${ }^{3}$.

Peri-implantitis was reported in three patients with poor oral hygiene, and a total of 20 dental implants failed (14.70\%). Implant failures occurred in three patients, and these rates were obtained without considering the replaced implants. The reasons of implant failure were peri-implantitis (2 patients) and lack of osseointegration (1 patient). Gingival hyperplasia around one implant due to lack of attached gingiva was reported. The failure rate was higher in maxillae. Meta-analysis of the probability of failure was $7 \%$ (95\% confidence interval [CI] 0\%-31\%) for maxillary implants and 2\% (95\% CI $0 \%$ $9 \%$ ) for mandibular implants. The value of $\mathrm{I}^{2}$ was $73 \%$ and $0 \%$ for maxillary and mandibular implants, respectively. The overall result for all $\mathrm{I}^{2}$ was $50.81 \%$ with a pooled random effect estimate of $3 \%(95 \%$ CI $0 \%-12 \%)$. A forest plot of the meta-analysis is provided in Fig. 2. None of the patients had healing problems after the placement of implants or bone graft. The mean and median follow-up after prosthetic rehabilitation was $5.16 \pm 5.08$ years and 4 years $(\mathrm{Q} 1=1, \mathrm{Q} 2=8.75)$, respectively. The follow-up time ranged between 1 years and 20 years.

\section{Discussion}

Periodontitis is an important manifestation of PLS and, in these patients, the conventional periodontal treatments usually cannot prevent the progressive attachment $\operatorname{loss}^{8,14}$. In addition to periodontal treatment, antimicrobial therapies including erythromycin, tetracycline, penicillin, and amoxicillinmetronidazole have been suggested. However, controversial

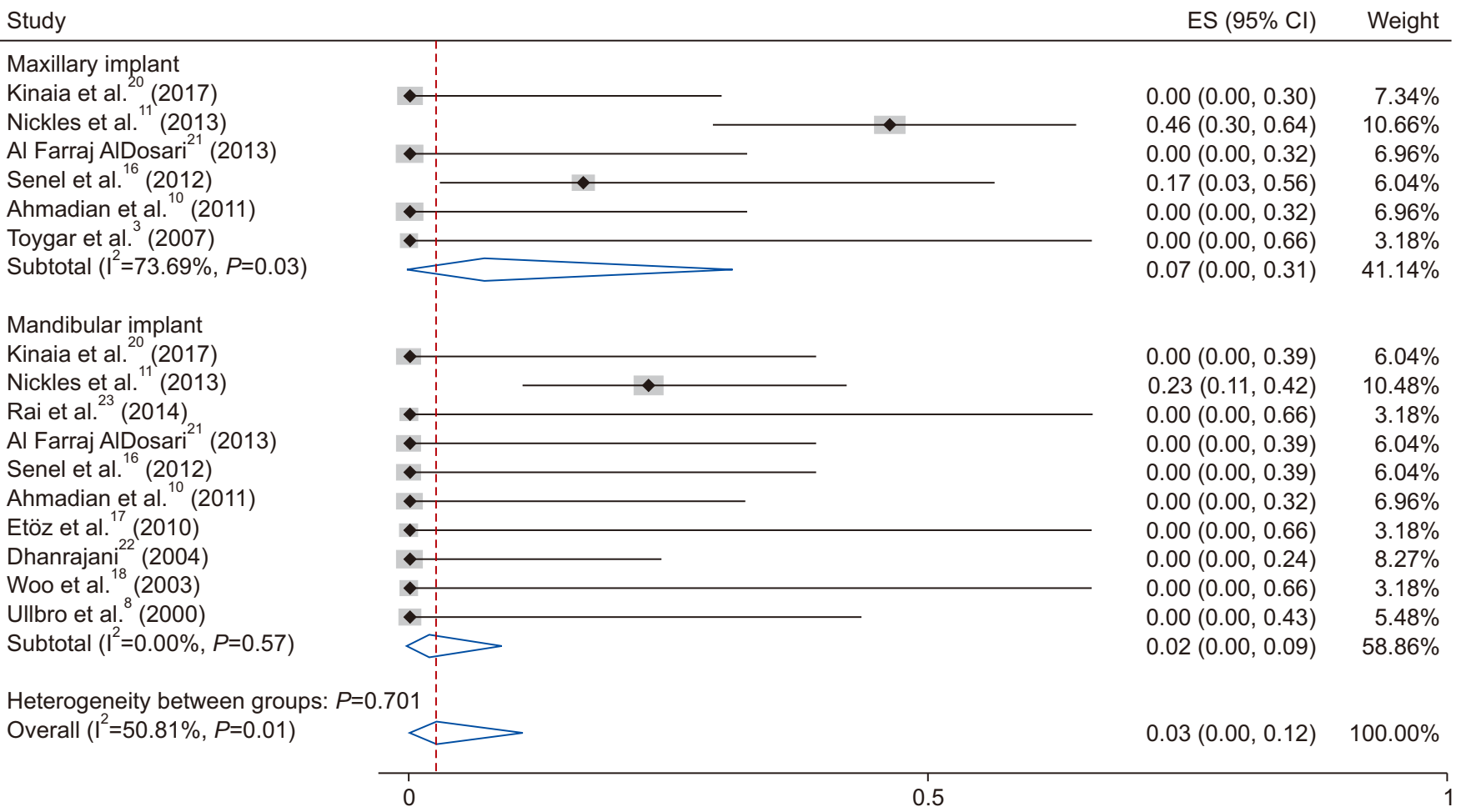

Fig. 2. Probability of implant failure based on maxillary and mandibular implant. (ES: effect size, Cl: confidence interval) Fazele Atarbashi-Moghadam et al: Oral rehabilitation of Papillon-Lefêvre syndrome patients by dental implants: a systematic review. J Korean Assoc Oral Maxillofac Surg 2020 
results have been reported ${ }^{15}$. Continuous attachment and tooth loss are present in some patients ${ }^{11,14}$. These patients experience partial or complete edentulism during their adolescence $^{8}$ and need oral reconstruction. Conventional complete denture or overdenture is the traditional prosthetic treatment for PLS patients, but this treatment causes esthetic and functional problems that lead patients to seek a more comprehensive treatment ${ }^{10}$. The chief patient complaints were the lack of stability and retention of their mandibular prosthesis ${ }^{8,16-18}$. Dental implants can provide the necessary support, stability, and retention for dental prostheses ${ }^{10}$ and are, therefore, helpful to PLS patients. The benefits of implants include not only enhanced prosthesis stability and retention, but also preservation of the supporting bone and prevention of further bone loss $^{8}$.

Extraction of all PLS patient primary teeth so that the patient experiences an edentulism period between primary and permanent dentition has been reported to be helpful ${ }^{9,14,19}$.

Although implants help patients with edentulism, lack of available bone for dental implant placement as the result of progressive periodontitis and/or continuous use of full dentures for many years is the major problem for these patients ${ }^{10,17,18}$. Therefore, an implant-based treatment plan for these patients is, in many cases, restricted to overdentures unless complicated pre-surgical bone augmentations are required $^{10}$. In this review, pre-surgical augmentation was reported in three patients. These included sinus augmentation, inferior alveolar nerve repositioning and guided bone regeneration (GBR) using extra-oral harvesting bone (calvaria and tibia) or bone substitute material ${ }^{3,10,20}$. Simultaneous GBR was also performed during implant placement if needed ${ }^{21}$. In all cases with bone augmentation, the healing period was normal and uneventful $1^{3,10,20,21}$.

To avoid these complicated and expensive procedures, implant placement between the mental foramina for fixed mandibular prosthesis could be the treatment of choice if sufficient bone is available in the anterior mandible ${ }^{8,22}$. Insertion of two implants in the anterior mandible was another treatment modality for an implant-supported removable overdenture $^{17,18,23}$. Etöz et al. ${ }^{17}$ used short dental implants for mandibular overdenture support. Their patient showed severe ridge atrophy so distraction osteogenesis had a potential for bone fracture ${ }^{17}$.

Osseointegration occurred successfully except for the early failure of one implant. For areas lacking soft and hard tissue, distraction osteogenesis is suggested ${ }^{3}$.

Another consideration for PLS patients is the age at edentu- lism. Most of the patients lost their teeth early; these patients lost most of their permanent teeth by 14 years 5 . However, dental implants act as ankylosed teeth and are contraindicated in teenagers and growing individuals ${ }^{24}$. Dental implants inserted in patients under the age of 18 led to infra-occlusal positioning of the maxillary dental implant. Insertion of dental implants in the anterior mandible encountered less complications ${ }^{1}$. Ullbro et al. ${ }^{8}$ suggested that dental implant complications in growing PLS patients was less important than bone preservation. Bohner et al. ${ }^{1}$ suggested that, whenever growing patients may benefit more from dental implants, the implantation can be performed cautiously; maintenance follow-ups and implant-supported prosthesis adjustments are required until growth cessation. However, in their systematic review only anterior region implant placements were considered ${ }^{1}$. Dental implant placement before the cessation of growth had been performed in patients with ectodermal dysplasia. A systematic review ${ }^{25}$ demonstrated that the rate of dental implants failure in these patients was relatively low (5.3\%-7.2\%).

Impaired immune systems of young PLS patients is another consideration ${ }^{11}$. PLS patient neutrophils are deficient in the ability to establish neutrophil extracellular traps (NET), and chemotactic velocity is also reduced in PLS patient neutrophils ${ }^{7}$. However, clinical evaluation and long-term followup of PLS patients have shown that the function of PMNs in PLS patients improves with age ${ }^{8,9}$. Tinanoff et al. ${ }^{9}$ reported normal PMN chemotaxis and adherence in their patient after 15 years follow-up (at age 24 years). Ullbro et al. ${ }^{8}$ tested the PMN chemotaxis and phagocytosis of their patient before implant placement (at age 25 years) which had improved to normal values. Therefore, the insertion of PLS patient dental implants at younger ages may result in the same inflammatory process as the one that occurs in the teeth. Based on these articles, the optimum age for implant placement in PLS patients is still unclear; and, if implant treatment is performed at an early age, immunological analysis is necessary. Most of the cases in this review received dental implant treatment after the age of 18 .

This systematic review assessed the results of dental implant treatment in PLS patients. The longest follow-up periods after implant placement reported in these studies were 20 years and 10 years ${ }^{11}$. Other extended follow-up periods included 9 years $^{22}, 4.5$ years $^{8}$, and 4 years ${ }^{10,11}$. In $40 \%$ of patients the implant follow-up time was 2 years or less.

Peri-implantitis occurred in three patients. Implant failure (19 implants) resulted in two of these patients. Poor oral hygiene and poor compliance with the maintenance program 
were reported as a probable cause of implant failure. Another implant failure occurred due to lack of osseointegration; implant replacement was successful. One implant showed gingival hyperplasia due to lack of attached gingiva. Poor oral hygiene and lack of regular attendance at recall visits were reported as important factors in occurrence of peri-implant diseases $^{26-28}$. These results emphasize that oral hygiene and compliance with follow-up programs have important roles in PLS patient implant success. The results of this study showed a higher rate of maxillary peri-implantitis and implant failure. (Table 1, Fig. 2) The data concerning the higher prevalence of maxillary peri-implantitis was heterogeneous and this relationship was not proven ${ }^{29}$.

More cases with long-term follow-up results are required for drawing definite conclusions about dental implant treatment modalities in PLS patients.

\section{ORCID}

Fazele Atarbashi-Moghadam, https://orcid.org/0000-00023499-2250

Saede Atarbashi-Moghadam, https://orcid.org/0000-00021731-2786

Setare Kazemifard, https://orcid.org/0000-0002-2892-5019 Soran Sijanivandi, https://orcid.org/0000-0003-4663-4423

Mahshid Namdari, https://orcid.org/0000-0002-7069-6977

\section{Author's Contributions}

F.A.M. and S.A.M. conceived the ideas. S.K., F.A.M., and S.S. collected the data. M.N. analyzed the data. F.A.M. and S.S. did the writing.

\section{Conflict of Interest}

No potential conflict of interest relevant to this article was reported.

\section{References}

1. Bohner L, Hanisch M, Kleinheinz J, Jung S. Dental implants in growing patients: a systematic review. Br J Oral Maxillofac Surg 2019;57:397-406. https://doi.org/10.1016/j.bjoms.2019.04.011

2. Heuberer S, Dvorak G, Mayer C, Watzek G, Zechner W. Dental implants are a viable alternative for compensating oligodontia in adolescents. Clin Oral Implants Res 2015;26:e22-7. https://doi. org/10.1111/clr.12323

3. Toygar HU, Kircelli C, Firat E, Guzeldemir E. Combined therapy in a patient with Papillon-Lefèvre syndrome: a 13-year followup. J Periodontol 2007;78:1819-24. https://doi.org/10.1902/ jop.2007.070004

4. Machado RA, Cuadra-Zelaya FJM, Martelli-Júnior H, Miranda RT, Casarin RCV, Corrêa MG, et al. Clinical and molecular analysis in Papillon-Lefèvre syndrome. Am J Med Genet A 2019;179:212431. https://doi.org/10.1002/ajmg.a.61285

5. Hart TC, Shapira L. Papillon-Lefèvre syndrome. Periodontol 2000 1994;6:88-100. https://doi.org/10.1111/j.1600-0757.1994.tb00029.x

6. Nagy N, Vályi P, Csoma Z, Sulák A, Tripolszki K, Farkas K, et al. CTSC and Papillon-Lefèvre syndrome: detection of recurrent mutations in Hungarian patients, a review of published variants and database update. Mol Genet Genomic Med 2014;2:217-28. https:// doi.org/10.1002/mgg3.61

7. Roberts H, White P, Dias I, McKaig S, Veeramachaneni R, Thakker N, et al. Characterization of neutrophil function in PapillonLefèvre syndrome. J Leukoc Biol 2016;100:433-44. https://doi. org/10.1189/jlb.5A1015-489R

8. Ullbro C, Crossner CG, Lundgren T, Stålblad PA, Renvert S. Osseointegrated implants in a patient with Papillon-Lefèvre syndrome. A 4 1/2-year follow up. J Clin Periodontol 2000;27:951-4. https://doi.org/10.1034/j.1600-051x.2000.027012951.x

9. Tinanoff N, Tempro P, Maderazo EG. Dental treatment of Papillon-Lefèvre syndrome: 15-year follow-up. J Clin Periodontol 1995;22:609-12. https://doi.org/10.1111/j.1600-051x.1995. tb00813.x

10. Ahmadian L, Monzavi A, Arbabi R, Hashemi HM. Full-mouth rehabilitation of an edentulous patient with Papillon-Lefèvre syndrome using dental implants: a clinical report. J Prosthodont 2011;20:643-8. https://doi.org/10.1111/j.1532-849X.2011.00768.x

11. Nickles K, Schacher B, Ratka-Krüger P, Krebs M, Eickholz P. Long-term results after treatment of periodontitis in patients with Papillon-Lefèvre syndrome: success and failure. J Clin Periodontol 2013;40:789-98. https://doi.org/10.1111/jcpe.12120

12. Moher D, Liberati A, Tetzlaff J, Altman DG; PRISMA Group. Preferred reporting items for systematic reviews and meta-analyses: the PRISMA statement. Ann Intern Med 2009;151:264-9, W64. https://doi.org/10.1371/journal.pmed.1000097

13. Riley DS, Barber MS, Kienle GS, Aronson JK, von Schoen-Angerer T, Tugwell P, et al. CARE guidelines for case reports: explanation and elaboration document. J Clin Epidemiol 2017;89:218-35. https://doi.org/10.1016/j.jclinepi.2017.04.026

14. De Vree H, Steenackers K, De Boever JA. Periodontal treatment of rapid progressive periodontitis in 2 siblings with Papillon-Lefèvre syndrome: 15-year follow-up. J Clin Periodontol 2000;27:354-60. https://doi.org/10.1034/j.1600-051x.2000.027005354.x

15. Pacheco JJ, Coelho C, Salazar F, Contreras A, Slots J, Velazco $\mathrm{CH}$. Treatment of Papillon-Lefèvre syndrome periodontitis. J Clin Periodontol 2002;29:370-4. https://doi.org/10.1034/j.1600051x.2002.290414.x

16. Senel FC, Altintas NY, Bagis B, Cankaya M, Pampu AA, Satiroglu I, et al. A 3-year follow-up of the rehabilitation of Papillon-Lefèvre syndrome by dental implants. J Oral Maxillofac Surg 2012;70:1637. https://doi.org/10.1016/j.joms.2011.03.058

17. Etöz OA, Ulu M, Kesim B. Treatment of patient with Papillon-Lefèvre syndrome with short dental implants: a case report. Implant Dent 2010;19:394-9. https://doi.org/10.1097/ ID.0b013e3181ed0798

18. Woo I, Brunner DP, Yamashita DD, Le BT. Dental implants in a young patient with Papillon-Lefèvre syndrome: a case report. Implant Dent 2003;12:140-4. https://doi.org/10.1097/01. id.0000041223.08656.a7

19. Wiebe CB, Häkkinen L, Putnins EE, Walsh P, Larjava HS. Successful periodontal maintenance of a case with Papillon-Lefèvre syndrome: 12-year follow-up and review of the literature. J Periodontol 2001;72:824-30. https://doi.org/10.1902/jop.2001.72.6.824

20. Kinaia BM, Hope K, Zuhaili A, Tulasne JF. Full-mouth rehabilita- 
tion with calvarium bone grafts and dental implants for a PapillonLefèvre syndrome patient: case report. Int J Oral Maxillofac Implants 2017;32:e259-64. https://doi.org/10.11607/jomi.6282

21. Al Farraj AlDosari A. Oral rehabilitation of a case of PapillonLefèvre syndrome with dental implants. Saudi Med J 2013;34:4247.

22. Dhanrajani PJ. Re: "Dental implants in a young patient with Papillon-Lefèvre syndrome: a case report" (Implant Dent. 2003;12(2):140-4). Implant Dent 2004;13:280. https://doi. org/10.1097/01.id.0000148561.03643.75

23. Rai R, Kumar A, Deshpande V. Papillon-Lefèvre syndrome - the prosthodontic management. J Pierre Fauchard Acad 2014;28:23-7. https://doi.org/10.1016/j.jpfa.2014.03.002

24. Oesterle LJ, Cronin RJ Jr, Ranly DM. Maxillary implants and the growing patient. Int J Oral Maxillofac Implants 1993;8:377-87.

25. Chrcanovic BR. Dental implants in patients with ectodermal dysplasia: a systematic review. J Craniomaxillofac Surg 2018;46:12117. https://doi.org/10.1016/j.jcms.2018.05.038

26. Cortellini S, Favril C, De Nutte M, Teughels W, Quirynen M. Patient compliance as a risk factor for the outcome of implant treatment. Periodontol 2000 2019;81:209-25. https://doi.org/10.1111/ prd. 12293
27. Lin CY, Chen Z, Pan WL, Wang HL. The effect of supportive care in preventing peri-implant diseases and implant loss: a systematic review and meta-analysis. Clin Oral Implants Res 2019;30:714-24. https://doi.org/10.1111/clr.13496

28. Atarbashi-Moghadam F, Atarbashi-Moghadam S, Namdari M, Shahrabi-Farahani S. Reactive oral lesions associated with dental implants. A systematic review. J Investig Clin Dent 2018;9:e12342. https://doi.org/10.1111/jicd.12342

29. Dreyer H, Grischke J, Tiede C, Eberhard J, Schweitzer A, Toikkanen SE, et al. Epidemiology and risk factors of peri-implantitis: a systematic review. J Periodontal Res 2018;53:657-81. https://doi. org/10.1111/jre. 12562

How to cite this article: Atarbashi-Moghadam F, AtarbashiMoghadam S, Kazemifard S, Sijanivandi S, Namdari M. Oral rehabilitation of Papillon-Lefèvre syndrome patients by dental implants: a systematic review. J Korean Assoc Oral Maxillofac Surg 2020;46:220-227. https://doi.org/10.5125/jkaoms.2020.46.4.220 